\title{
Design suggestions for an mHealth app to facilitate communication between pharmacists and the Deaf: perspective of the Deaf community (HEARD Project)
}

\author{
Sabrina Anne Jacob ${ }^{1,2}$, Elizabeth Yie-Chuen Chong ${ }^{1}$, Soo Leng Goh ${ }^{3}$, Uma Devi Palanisamy ${ }^{4}$ \\ ${ }^{1}$ School of Pharmacy, Monash University Malaysia, Jalan Lagoon Selatan, Selangor, Malaysia; ${ }^{2}$ Strathclyde Institute of Pharmacy and Biomedical \\ Sciences, University of Strathclyde, Glasgow, Scotland; ${ }^{3}$ Malaysian Federation of the Deaf, Puchong, Selangor, Malaysia; ${ }^{4}$ Jeffrey Cheah School of \\ Medicine and Health Sciences, Monash University Malaysia, Jalan Lagoon Selatan, Selangor, Malaysia \\ Contributions: (I) Conception and design: SA Jacob, UD Palanisamy, SL Goh; (II) Administrative support: None; (III) Provision of study materials or \\ patients: None; (IV) Collection and assembly of data: SL Goh; (V) Data analysis and interpretation: SL Goh, SA Jacob, EYC Chong; (VI) Manuscript \\ writing: All authors; (VII) Final approval of manuscript: All authors. \\ Correspondence to: Sabrina Anne Jacob. School of Pharmacy, Monash University Malaysia, Jalan Lagoon Selatan, 47500 Sunway, Selangor, Malaysia. \\ Email: sabrina.jacob@strath.ac.uk.
}

\begin{abstract}
Background: Deaf and hard-of-hearing (DHH) patients have trouble communicating with community pharmacists and accessing the healthcare system. This study explored the views on a proposed mobile health (mHealth) app in terms of design and features, that will be able to bridge the communication gap between community pharmacists and DHH patients.

Methods: A community-based participatory research method was utilized. Two focus group discussions (FGDs) were conducted in Malaysian sign language (BIM) with a total of $10 \mathrm{DHH}$ individuals. Respondents were recruited using purposive sampling. Video-recordings were transcribed and analyzed using a thematic approach.

Results: Two themes emerged: (I) challenges and scepticism of the healthcare system; and (II) features of the mHealth app. Respondents expressed fears and concerns about accessing healthcare services, and stressed on the need for sign language interpreters. There were also concerns about data privacy and security. With regard to app features, the majority preferred videos instead of text to convey information about their disease and medication, due to their lower literacy levels.

Conclusions: For an mHealth app to be effective, app designers must ensure the app is individualised according to the cultural and linguistic diversity of the target audience. Pharmacists should also educate patients on the potential benefits of the app in terms of assisting patients with their medicine-taking.
\end{abstract}

Keywords: Deaf; community pharmacist; mHealth; pharmaceutical care; communication

Received: 02 October 2019; Accepted: 15 January 2020; Published: 20 April 2021.

doi: 10.21037/mhealth.2020.01.04

View this article at: http://dx.doi.org/10.21037/mhealth.2020.01.04

\section{Introduction}

Deaf and hard-of-hearing (DHH) individuals utilize healthcare services differently from the general population, and often delay or avoid seeking medical care, or selfmedicate, due to communication barriers faced with healthcare professionals $(1,2)$. Deaf with an uppercase 'd' refers to a group that shares a cultural identity and a common language-sign language (3). Indeed, when communicating with the Deaf, only sign language can precisely deliver information (4).

Studies have shown that most healthcare professionals are linguistically and culturally unprepared to serve the needs of the DHH $(1,5,6)$, which has been attributed to their lack of knowledge and awareness of Deaf culture (7). 
There is also a misconception that sign language is a visual representation of the spoken language $(8,9)$. This has then led to a reliance on lip-reading and using written text to communicate with the $\mathrm{DHH}$, which has only perpetuated the communication divide (5). A focus group discussion (FGD) was conducted to determine the communication barriers and needs of the $\mathrm{DHH}$ when seeking pharmaceutical care. Although the DHH acknowledge written text as a mode of communication, they often found the English used by pharmacists too complex to comprehend (5). Indeed, instructions on medication use are usually given either verbally or in written form by the pharmacist-methods which are not viable for $\mathrm{DHH}$ individuals (4). Health education materials are also usually above the recommended sixth-grade level, while written communication with the Deaf should generally be at fourthgrade and below $(6,10,11)$.

A number of tools are currently available to assist the Deaf with communication. The Deaf can use teletypewriters (TTY) to type their messages using a keyboard through telephone lines. A relay operator then conveys the typed messages using either text or voice format through the telephone (1). Contrary to TTY, video relay services (VRS) use sign language interpreters (SLIs) as relay operators to assist communication between the Deaf and hearing person on a computer screen through linked video cameras (1). TTY and VRS, while effective, result in prolonged communication time (12), and require skilled manpower (13). Table 1 provides further details on tools used by the Deaf to communicate, as well as Deaf-related terms.

Mobile health (mHealth) is a tool with mobile functions and wireless technologies that has shown success in health promotion and monitoring activities, as well as a communication tool for linguistically and culturally diverse groups $(16,17)$. A number of apps designed to assist communication between healthcare professionals and Deaf individuals exist in South Africa, Germany, and the United States. These apps rely on either pre-recorded videos based on pre-defined phrases, or transcribing live conversations to text in languages such as English or German (18-20).

Such an app will prove beneficial in Malaysia, which has an estimated 55,000 Deaf individuals. The Deaf community in Malaysia currently face a shortage in SLIs, with a ratio of one interpreter to 1,000 Deaf individuals (21). Healthcare professionals in Malaysia are also not obligated by law to provide an SLI during patient visits, leading to fears of misdiagnosis (1), and apprehension in utilising the healthcare system (22). In addition, in a recent pilot study with members of the Malaysian Federation of the Deaf (MFD) assessing their utilization of community pharmacy services, Deaf participants highlighted that current health services were not catered to the needs of the Deaf, and communication with community pharmacists was less than optimal (23).

According to a nationwide survey, more than $70 \%$ of Malaysian consumers purchased their medications from community pharmacies (24). There is also still an absence of dispensing separation in Malaysia (25). Many of the obstacles experienced by the DHH take on a greater salience in emerging and developing countries where pharmacists have limited time due to patient load (4). As noted, Malaysian sign language (BIM) is identifiably distinct from other sign languages used in different countries, and is heavily influenced by Malaysian culture (15). As such, mHealth app developers must be cognizant of this when creating apps that rely on sign language. We plan to design and develop an app to facilitate communication between community pharmacists and individuals who are Deaf. Thus, FGDs were conducted with Deaf participants to explore their views on the proposed mHealth app in terms of design and features.

\section{Methods}

The findings of this qualitative study are reported according to the Consolidated Criteria for Reporting Qualitative Studies (COREQ) (26).

\section{Research team and reflexivity}

The research team was made up of a pharmacy-qualified academic (SA Jacob), an undergraduate pharmacy research assistant (EY Chong), an academic in biomedical sciences (UD Palanisamy), and a member of the MFD (SL Goh). Three members of the team were experienced in qualitative research and studies focusing on the healthcare needs of the Deaf (EY Chong, UD Palanisamy and SA Jacob). Two members had collaborated with the MFD to coordinate service learning placements for pharmacy students (UD Palanisamy and SA Jacob). SL Goh was a native signer who worked with the MFD and has done research in social theory, sociological theory and developmental psychology. Three of the researchers had no prior interaction with the participants of the study (EY Chong, UD Palanisamy and SA Jacob), and none of the researchers were involved in the care of the participants. A community-based participatory 
Table 1 Deaf-related terms and definitions*

\begin{tabular}{|c|c|}
\hline Term & Definition \\
\hline $\begin{array}{l}\text { Sign language interpreter } \\
\text { (SLI) }\end{array}$ & A person trained in translating between a spoken and a signed language \\
\hline Video relay services (VRS) & $\begin{array}{l}\text { Uses SLIs to assist communication between a Deaf person who uses a 'videophone' to communicate in sign } \\
\text { language, and a hearing person who uses a standard telephone; on a computer screen through linked video } \\
\text { cameras (1) }\end{array}$ \\
\hline $\begin{array}{l}\text { Video remote interpreting } \\
\text { (VRI) }\end{array}$ & $\begin{array}{l}\text { Uses video conferencing technology where SLIs are remote or off site, and communicate with the Deaf person } \\
\text { via video in sign language, but with the hearing person in audio }\end{array}$ \\
\hline Videophone (VP) & $\begin{array}{l}\text { Deaf people who use a VRS have a phone number designated "VP". If the person they wish to call also has a } \\
\text { VP, they can call the person directly and have a video conversation in sign language. If the person they wish to } \\
\text { call uses a voice telephone, the call is routed through a VRS (1) }\end{array}$ \\
\hline
\end{tabular}

${ }^{*}$ This table is adapted from Patient Preference and Adherence Chong 201913 195-207 with permission of the original publisher Dove Medical Press Ltd. (9).

research method was adopted, where members of the Deaf community were involved in the development of the research questions and conduct of the FGDs (27). GSL conducted both FGDs. Participants were given information on the credentials of the researchers prior to the start of the FGDs, and were told the objectives of the study.

\section{Study design}

Focus groups employing a grounded-theory approach were adopted. Purposive sampling was employed to recruit people who were Deaf or $\mathrm{HoH}$, aged 18 years and above. An advertisement to participate was posted in the MFD, and those interested were told to get in touch with the researchers. Potential participants were also approached in person by GSL.

Both FGDs were conducted in MFD. The sessions were video-recorded, and field notes were also taken to capture key points. Participants were requested to provide some demographic details at the end of the FGDs, and were given gift vouchers amounting to USD5 to thank them for their participation. The interview guide was developed based on the study objectives, review of the literature, as well as information needed by the app developers (supplementary). Face and content validation were done by experts in qualitative research, members of the Deaf community, and experts in app-design and development. Analysis revealed saturation of themes and as such no further interviews were undertaken.

\section{Data transcription and analysis}

Recorded interviews were transcribed by SL Goh, and deidentified prior to analysis. The transcribed data was then verified by another researcher (EY Chong). Results were imported into QSR International's NVivo 11 Software, and thematic analysis was performed on the transcripts by two researchers (SA Jacob and EY Chong), guided by Braun and Clarke's six phase approach to coding: (I) data familiarization; (II) initial code generation; (III) theme searching; (IV) reviewing of themes; (V) defining and naming themes; and (VI) producing the report (28). 
Table 2 Characteristics of respondents

\begin{tabular}{|c|c|}
\hline Characteristics* $^{*}$ & N (\%) \\
\hline \multicolumn{2}{|c|}{ Age diagnosed as deaf } \\
\hline Since birth & $3(37.5)$ \\
\hline As a baby & $2(25.0)$ \\
\hline 2 years old & $1(12.5)$ \\
\hline 5 years old & $1(12.5)$ \\
\hline 6 years old & $1(12.5)$ \\
\hline \multicolumn{2}{|c|}{ Highest level of education } \\
\hline Primary & $3(37.5)$ \\
\hline Secondary & $1(12.5)$ \\
\hline Certificate & $3(37.5)$ \\
\hline Diploma & $1(12.5)$ \\
\hline \multicolumn{2}{|c|}{ Languages they can read } \\
\hline BM & $8(100)$ \\
\hline English & $2(25.0)$ \\
\hline \multicolumn{2}{|c|}{ Languages they can speak in } \\
\hline BM & $7(87.5)$ \\
\hline English & $2(25.0)$ \\
\hline \multicolumn{2}{|c|}{ Languages they can understand } \\
\hline BM & $8(100)$ \\
\hline BIM & $8(100)$ \\
\hline
\end{tabular}

*Data only available for eight respondents. BM, Bahasa

Malaysia; BIM, Malaysian sign language.

\section{Ethical considerations}

This study was approved by the Monash University Human Research Ethics Committee (CF16/18-2016000007). The explanatory statement was given out to all participants and the contents were communicated to them using BIM, which included information on study objectives and possible risks. Signed consent forms were then obtained from all participants. The authors are accountable for all aspects of the work.

\section{Results}

Two FGDs were conducted, with five participants in each group. Of the 10, only one participant was male, and two declined to provide further demographic details. The remaining eight owned smartphones with Android operating systems, and had an age range of 25 to 47 years. They were currently not using smartphone medical apps but were willing to try them. Further details are summarized in Table 2. Responses from all 10 participants were included in the thematic analysis, which revealed two themes: (I) challenges and scepticism of the healthcare system; and (II) features of the mHealth app, and are described below.

\section{Theme 1: challenges and scepticism of the healthcare system}

Seven participants agreed that communicating with pharmacists was challenging, and that they had experienced miscommunication with pharmacists. Three expressed fear and anxiety when visiting a pharmacy, especially when alone, and resorted to taking along a family member, friend, or SLI to aid communication. When alone, they were obliged to communicate in writing with the pharmacist, but noted that even then, miscommunication still occurred. In view of these challenges with communication, participants were asked if they would prefer to use a voice-to-text conversion service via the app to help them communicate better. Six participants acknowledged that since not all Deaf individuals were literate, or could not fully understand the meaning of what they had read, the voice-to-text conversion may not be helpful. For that reason, some suggested providing sign language interpretation alongside the voiceto-text conversion. The SLI would then be able to explain the instructions to them.

Participants were asked about Grab \& Go, a feature of the app where users are able to pre-order medications through the in-built catalogue and pick them up instore. Most found it enticing, however, a few participants voiced fears or concerns that the wrong medications would be packed. Thus, some participants commented that pharmacists must ensure the correct medications are packed to avoid the hassle of returning to the pharmacy for rectification. There were also concerns if the medicines purchased through the app would be genuine. As for the delivery service, five of the participants said they would not utilise it as they were afraid the wrong medication might be sent. Participants were asked about a comparison tool, where users can compare different brands by price, side effects, tablet size, and so forth prior to purchasing medications. However, the majority preferred to seek the pharmacists' expertise personally rather than using the app. When purchasing medicine, two participants said that price is not the main criteria as long as the medicine is helping. 
One participant also highlighted that she was sometimes unsure if the medication was suitable for her.

Opinions among participants were divided with regard to the disclosure of personal information such as medical history and allergies in the app. Although one group found no problem in providing personal information, the other was uncertain. Some felt that it was acceptable to share their personal information on the app as long as it was only viewed by doctors and pharmacists. A similar concern was voiced when participants were asked if they preferred filling up forms through the app or in person. One participant said that he would prefer to fill it in person and pass it to the person at the counter himself, as he was afraid his personal data may be divulged to non-healthcare parties.

\section{Theme 2: features of the mHealth app}

Besides providing text descriptions regarding medication queries, participants felt providing video directions was necessary as not all Deaf individuals were literate. Similarly, participants noted a preference for visuals in video form accompanied by subtitles, with a suggestion for captions in multiple languages. There was also a suggestion to include pictures of medicines. Other than that, in-app notifications, which are alerts sent from the app to the mobile device to remind them when to collect their medication, were preferred compared to conventional text messages, as they were perceived to be more convenient. When asked if they would prefer a quick chat tool to an app, participants were unanimous that it would be better to communicate with a pharmacist face to face to seek clarification.

Other proposed features of the app that participants found helpful were the online booking service within the app to schedule appointments as they felt it would be time-saving, and using the app to provide details prior to their visit. With regard to pricing, the consensus was that the app should be free, however one participant mentioned considering it if the cost was minimal. Six participants requested for the app to include sign language interpreting services.

\section{Discussion}

Findings from this study highlighted the challenges the Deaf face in communicating with community pharmacists, as well as their fears and mistrust of the healthcare system. While participants welcomed the idea of an app, there were concerns about privacy and data security.

Our study revealed that while patients were positive regarding certain aspects of the app such as the scheduling service, Grab \& Go, and using the app to provide details prior to a visit; they preferred consulting with the pharmacists on issues related specifically to their medication instead of solely relying on information provided via the app. Indeed studies have found that a combination of written and verbal information was preferred by patients $(29,30)$, and is more effective at increasing the knowledge level of patients (31). This could be due to the fact that patients struggle to comprehend and use written medical information due to lower health literacy levels (32)a situation which will be compounded in the Deaf. This highlights that the mHealth app should not be viewed as a substitute for counselling services provided by pharmacists, but instead as a tool to enhance the provision of medical information to patients.

There was also some scepticism with regard to the suitability of the medicine, while some participants mentioned that cost was not an issue as long as the medicine actually helped them. These doubts could stem from two main factors: (I) miscommunication with pharmacists which has led to nonadherence to medications $(5,33)$. Both lower health literacy levels and being Deaf are also barriers to adherence (33), which translate to poorer health outcomes $(5,33)$. This has then led them to erroneously assume that the medicine was not suitable for them; and (II) cultural factors which have resulted in cynicism about the use of Western medicine, which is perceived to be harmful and not as safe as Eastern medication (34-37). Malaysia is a multicultural society made of Malays (60\%), Chinese (>20\%), Indians $(\sim 10 \%)$, and other native ethnic groups; with each group having their own religious beliefs which have been shown to impact medication adherence (38). The onus is thus on the pharmacist to firstly train themselves on how to communicate with the Deaf, address the culturallyassociated negative attitudes and beliefs they hold with regard to medication, and educate them on the importance of adherence.

Participants also highlighted data security concerns, similar with a survey of approximately 12,000 adult patients, where more than $50 \%$ had misgivings about using health IT due to concerns about data privacy (39). To overcome this, pharmacists should engage with patients and address these privacy concerns, educating them on the security features related to their personal information, how their personal data and health information is protected under the Personal Data Protection Act 2010 in Malaysia, and the importance of sharing this personal information with the 
pharmacists (40).

The plight of Deaf patients in Malaysia is similar to that in other countries: limited literacy levels, infrequent contact with health care providers who know their language and culture, limited information available in sign language, and the frequent necessity of using family and friends as interpreters (6). The reliance on a family member or friend has resulted in privacy concerns, and the patient being left out of the discussion most of the time - while writing down everything was seen as cumbersome. Thus, patients have reported preferring SLIs, as this eased the communication process $(11,41,42)$, similar with that professed by the majority of participants in this study. Being accompanied by someone knowledgeable in BIM also seemed to alleviate the anxiety patients faced when visiting the pharmacy.

Malaysia has satisfactory wireless broadband coverage throughout the country, and is one of the top countries to show a steep increase in smartphone ownership from 31\% in 2013 to $65 \%$ in 2016 (43). Indeed the majority of the participants in this study owned smartphones. As such, we believe that such an app will be well-received by the majority of the Deaf community in Malaysia. Findings from this study will be combined with the views received from community pharmacists with regard to app design, to ensure both parties are able to fully and effectively utilise the mHealth app.

\section{Limitations}

The study had a few limitations. For the purpose of analysis, data were transcribed from their source language. In the case of BIM, this equates to translation given that BIM has no written form. As a result, contexts and meanings may have been lost. This also means that no 'quotes' are available to be presented in the manuscript. The majority of participants were females, which might not represent the true perspective of all DHH patients in Malaysia. Indeed, it has been found that DHH women face more complex challenges accessing the healthcare system compared to men $(1,6)$, thus readers should interpret the findings in light of this. Although we only had 10 participants, data obtained here will be combined with an FGD involving community pharmacists (9), to inform the design of the mHealth app.

\section{Conclusions}

The FGD revealed that members of the Deaf community had some scepticism with a sole reliance on the mHealth app, and prefer to have a pharmacist as well as SLI present when it involved drug-related matters. We believe the app should be used by the pharmacist to enhance the patient counselling sessions with the Deaf. Pharmacists must also learn how to communicate with the Deaf and educate patients as to how the app could potentially help them in their medicine-taking.

\section{Acknowledgments}

The authors of the paper would like to thank the members of Malaysian Federation of the Deaf who gave their time to take part in this study.

Funding: This work was supported by the Health and Wellbeing Cluster, Global Asia in the 21st Century (GA21) at Monash University Malaysia.

\section{Footnote}

Conflicts of Interest: All authors have completed the ICMJE uniform disclosure form (available at http://dx.doi. org/10.21037/mhealth.2020.01.04). Dr. SAJ has a patent DITE (TM2018019083) issued. Dr. UDP has a patent DITE (TM2018019083) issued. The other authors have no conflicts of interest to declare.

Ethical Statement: The authors are accountable for all aspects of the work in ensuring that questions related to the accuracy or integrity of any part of the work are appropriately investigated and resolved. The study was conducted in accordance with the Declaration of Helsinki (as revised in 2013). This study was approved by the Monash University Human Research Ethics Committee (CF16/18-2016000007). Signed consent forms were obtained from all participants.

Open Access Statement: This is an Open Access article distributed in accordance with the Creative Commons Attribution-NonCommercial-NoDerivs 4.0 International License (CC BY-NC-ND 4.0), which permits the noncommercial replication and distribution of the article with the strict proviso that no changes or edits are made and the original work is properly cited (including links to both the formal publication through the relevant DOI and the license). See: https://creativecommons.org/licenses/by-nc-nd/4.0/.

\section{References}

1. Steinberg AG, Barnett S, Meador HE, et al. Health care 
system accessibility: experiences and perceptions of Deaf people. J Gen Intern Med 2006;21:260-6.

2. Drainoni ML, Lee-Hood E, Tobias C, et al. Crossdisability experiences of barriers to health-care access. Journal of Disability Policy Studies 2006;17:101-15.

3. Padden C, Humphries T. Deaf In America: Voices from a Culture. USA: Harvard University Press, 1988.

4. Motlhabi MB, Tucker WD, Parker MB, et al. Improving usability and correctness of a mobile tool to help a Deaf person with pharmaceutical instruction. In 4th Annual Symposium on Computing for Development (ACM DEV4); Cape Town, South Africa: University of the Western Cape Research Repository, 2013.

5. Ferguson M, Liu M. Communication needs of patients with altered hearing ability: informing pharmacists' patient care services through focus groups. J Am Pharm Assoc (2003) 2015;55:153-60.

6. Kuenburg A, Fellinger P, Fellinger J. Health care access among Deaf people. J Deaf Stud Deaf Educ 2016;21:1-10.

7. Hoang L, LaHousse S, Nakaji M, et al. Assessing Deaf Cultural Competency of Physicians and Medical Students. J Cancer Educ 2011;26:175-82.

8. McKee MM, Paasche-Orlow MK, Winters PC, et al. Assessing Health Literacy in Deaf American Sign Language Users. J Health Commun 2015;20 Suppl 2:92-100.

9. Chong EYC, Palanisamy UD, Jacob SA. A qualitative study on the design and development of an mHealth app to facilitate communication with the Deaf community: perspective of community pharmacists. Patient Prefer Adherence 2019;13:195-207.

10. Neuhauser L, Ivey SL, Huang D, et al. Availability and readability of emergency preparedness materials for Deaf and Hard-of-Hearing and older adult populations: issues and assessments. PLoS One 2013;8:e55614.

11. Meador HE, Zazove P. Health care interactions with Deaf culture. J Am Board Fam Pract 2005;18:218-22.

12. Vincent C, Bergeron F, Hotton M, et al. Message transmission efficiency through five telecommunication technologies for signing deaf users. Assist Technol 2010;22:141-51.

13. Wilson JAB, Schild S. Provision of mental health care services to deaf individuals using telehealth. Prof Psychol Res Pr 2014;45:324-31.

14. Smith RJH, Bale JF, White KR. Sensorineural hearing loss in children. Lancet 2005;365:879-90.

15. World Federation of the Deaf. Sign Language. In: Sign Language - WFD I World Federation of the Deaf. WFD
I World Federation of the Deaf. 2016. Available online: https://wfdeaf.org/human-rights/crpd/sign-language

16. Agarwal S, LeFevre AE, Lee J, et al. Guidelines for reporting of health interventions using mobile phones: mobile health (mHealth) evidence reporting and assessment (mERA) checklist. BMJ 2016;352:1174.

17. Kreps GL. The relevance of health literacy to mHealth. Stud Health Technol Inform 2017;240:347-55.

18. Chininthorn P, Glaser M, Tucker WD, et al. Exploration of Deaf people's health information sources and techniques for information delivery in Cape Town: a qualitative study for the design and development of a mobile health app. JMIR Human Factors 2016;3:e28.

19. iSignIT. App Synopsis. iSignIT, Germany. 2013. Available online: http://isignit.weebly.com/app-synopsis.html. Accessed 30 June 2017.

20. Ava. Ava For Professionals. Ava, United States of America. 2016. Available online: https://www.ava.me/about/. Accessed 30 June 2017.

21. Murad D. MFD: Massive shortage of sign language interpreters. Star Newspaper. 20 September 2013.

22. Kuenburg A, Fellinger P, Fellinger J. Health Care Access Among Deaf People. Journal of Deaf Studies and Deaf Education 2016;21:1-10.

23. Jacob SA, Chin JR, Ying Qi T, et al. The needs of the Deaf and hard of hearing when seeking pharmaceutical care. Res Social Adm Pharm 2016;12:664-5.

24. Mak WY. A snapshot of community pharmacy in Malaysia. MIMS, Malaysia. 2016. Available online: https://today. mims.com/a-snapshot-of-community-pharmacy-inmalaysia. Accessed 11 July 2018.

25. Hassali MA, Saleem F, Aljadhey H. Public Perceptions towards Implementation of Dispensing Separation: Results from a Cross Sectional Analysis. J Pharma Care Health Sys 2015;2:143.

26. Tong A, Sainsbury P, Craig J. Consolidated criteria for reporting qualitative research (COREQ): a 32-item checklist for interviews and focus groups. Int J Qual Health Care 2007;19:349-57.

27. McKee M, Thew D, Starr M, et al. Engaging the Deaf American Sign Language Community: Lessons From a Community-Based Participatory Research Center. Prog Community Health Partnersh 2012;6:321-9.

28. Braun V, Clarke V. Using thematic analysis in psychology. Qualitative Research in Psychology 2006;3:77-101.

29. Harvey JL, Plumridge RJ. Comparative Attitudes to Verbal and Written Medication Information among Hospital Outpatients. DICP 1991;25:925-8. 
30. Culbertson VL, Arthur TG, Rhodes PJ, et al. Consumer Preferences for Verbal and Written Medication Information. Drug Intell Clin Pharm 1988;22:390-6.

31. Johnson A, Sandford J. Written and verbal information versus verbal information only for patients being discharged from acute hospital settings to home: systematic review. Health Educ Res 2005;20:423-9.

32. Shrank WH, Avorn J. Educating Patients About Their Medications: The Potential And Limitations Of Written Drug Information. Health Affairs 2007;26:731-40.

33. McNary A. Avoiding risk with nonadherent patients. Innov Clin Neurosci 2015;12:37-40.

34. Ariff KM, Beng KS. Cultural health beliefs in a rural family practice: A Malaysian perspective. Aust J Rural Health 2006;14:2-8.

35. Risso-Gill I, Balabanova D, Majid F, et al. Understanding the modifiable health systems barriers to hypertension management in Malaysia: a multi-method health systems appraisal approach. BMC Health Serv Res 2015;15:254.

36. Mehmood KT, Anne JS. Brief review of complementary and alternative medicine use among Malaysian women with breast cancer. Journal of Pharmacy Practice and Research 2017;47:147-52.

37. Islahudin F, Shahdan IA, Mohamad-Samuri S. Association between belief and attitude toward preference of

doi: $10.21037 /$ mhealth.2020.01.04

Cite this article as: Jacob SA, Chong EY, Goh SL, Palanisamy UD. Design suggestions for a mHealth app to facilitate communication between pharmacists and the Deaf: perspective of the Deaf community (HEARD Project). mHealth 2021;7:29. complementary alternative medicine use. Patient Prefer Adherence 2017;11:913-8.

38. Hatah E, Lim KP, Ali AM, et al. The influence of cultural and religious orientations on social support and its potential impact on medication adherence. Patient Prefer Adherence 2015;9:589-96.

39. Heath S. Privacy issues, distrust keep patients from health IT engagement. Patient Engagement HIT 2017. Available online: https://patientengagementhit.com/news/privacyissues-distrust-keep-patients-from-health-it-engagement

40. Abdelhamid M, Gaia J, Sanders GL. Putting the focus back on the patient: how privacy concerns affect personal health information sharing intentions. J Med Internet Res 2017;19:e169.

41. Dickson M, Magowan R Meeting Deaf patients' communication needs. Nursing Times 2014;110:12-5.

42 Chaveiro N, Porto CC, Barbosa MA. The relation between Deaf patients and the doctor. Braz J Otorhinolaryngol 2009; 75:147-50.

43. Poushter J. Smartphone ownership rates skyrocket in many emerging economies, but digital divide remains. Pew Research Centre, Washington. 2016. Available online: http://www.pewglobal.org/2016/02/22/smartphoneownership-rates-skyrocket-in-many-emerging-economiesbut-digital-divide-remains/. Accessed 4 March 2018. 


\section{Appendix 1: questions for FGD}

1. Will the cost be a factor when purchasing apps?

2. Are location-based services that provide nearby deaffriendly pharmacies vital?

3. Are you willing to provide personal information, like medical history, allergies, etc., in the app to create a profile?

4. Do you base your medication use through web search? If so, would you prefer seeking advice through a video call from a professional instead?

5. Do you prefer to fill up forms through apps or in person?

6. Do you think it will ease the process if you fill forms prior your visit?

7. Do you prefer an in-app notification or a personal text from the pharmacist for the collection of your medication?

8. When uncertain, would you prefer a text description or image/video directions?

9. Will a vibrating device/pager notify you when the pharmacist is ready to see you?

10. Has miscommunication with the pharmacist led you to a wrong assignment in medication? If so, do you think a voice-to-text translation service would have prevented that incident from happening?

11. Would you utilize a booking service to schedule, reschedule and cancel appointments?

12. Will a Grab and Go service ease your visit to the pharmacist? Note* Purchase medications in-app and collect from the directed pharmacy.

13. Would you take the time to provide feedback/reviews on medications that may eventually help others? If not, would you still not do it if you were rewarded after?

14. When uncertain, would you utilize a quick chat tool on an app?

15. Would you utilize a click and compare tool when purchasing medication?

16. What sets you back from using in-app services?

17. Are visuals a key factor even when the content is the same?

18. Would you participate in community-based interaction? For example, support groups, etc.

19. Would you pay for delivery services? (One time/weekly/ monthly plan) 